\title{
The Role of Activity, Scan Duration and Patient's Body Mass Index in the Optimization of 18FDG Imaging Protocols on a TOF-PET/CT Scanner
}

\section{Roberta Matheoud ( $\sim$ roberta.matheoud@maggioreosp.novara.it)}

Azienda Ospedaliero-Universitaria Maggiore della Carita https://orcid.org/0000-0002-4783-0744

\section{Naema Al-Maymani}

ICTP: Abdus Salam International Centre for Theoretical Physics

\section{Alessia Oldani}

UPO

\section{Gian Mauro Sacchetti}

Azienda Ospedaliero-Universitaria Maggiore della Carità: Azienda Ospedaliero Universitaria Maggiore della Carita

\section{Marco Brambilla}

Azienda Ospedaliero-Universitaria Maggiore della Carità: Azienda Ospedaliero Universitaria Maggiore della Carita

\section{Alessandro Carriero}

Azienda Ospedaliero-Universitaria Maggiore della Carità: Azienda Ospedaliero Universitaria Maggiore della Carita

\section{Original research}

Keywords: Positron emission tomography, time-of flight, 18FDG, Contrast-to-noise ratio, optimization, image quality

Posted Date: December 31st, 2020

DOl: https://doi.org/10.21203/rs.3.rs-136005/v1

License: (c) (i) This work is licensed under a Creative Commons Attribution 4.0 International License. Read Full License

Version of Record: A version of this preprint was published at EJNMMI Physics on April 6th, 2021. See the published version at https://doi.org/10.1186/s40658-021-00380-9. 
1 The role of activity, scan duration and patient's body mass index in the optimization of 18FDG imaging protocols on a TOF-PET/CT scanner

3

4 Roberta Matheoud ${ }^{1}$, Naema Al-Maymani ${ }^{2,3}$, Alessia Oldani ${ }^{4}$, Gian Mauro Sacchetti ${ }^{5}$, Marco $5 \quad$ Brambilla $^{1}$, Alessandro Carriero $^{6}$

$7{ }^{1}$ Medical Physics Department, Azienda Ospedaliero-Universitaria Maggiore della Carità, C.so 8 Mazzini 18, 28100 Novara, Italy,

$9 \quad{ }^{2}$ Department of Physics, University of Trieste, Via Valerio 2, 34127 Trieste, Italy

3 Abdus Salam International Centre for Theoretical Physics (ICTP), Strada Costiera 11, 34151

11 Trieste, Italy

${ }^{4}$ Università del Piemonte Orientale, School of Medicine, V. Solaroli 17, 28100 Novara

${ }^{5}$ Nuclear Medicine Department, Azienda Ospedaliero-Universitaria Maggiore della Carità, C.so

Mazzini 18, 28100 Novara, Italy

${ }^{6}$ Radiology Department, Azienda Ospedaliero-Universitaria Maggiore della Carità, C.so Mazzini 18, 28100 Novara, Italy

Corresponding author:

22 Roberta Matheoud, $\mathrm{PhD}$

23 Medical Physics Department, Azienda Ospedaliero-Universitaria Maggiore della Carità

C.so Mazzini, 18 - 28100 Novara, Italy 
2 Background: Time-of-flight (TOF) PET technology determines a reduction in the noise and improves the reconstructed image quality in low counts acquisitions, such as in overweight patients, allowing a reduction of administered activity and/or imaging time. However, international guidelines and recommendations on ${ }^{18}$ F-fluoro-2-deoxyglucose (FDG) activity administration scheme are old or 6 only partially account for TOF technology and advanced reconstruction modalities.

The aim of this study was to optimize FDG whole-body studies on a TOF PET/CT scanner by using a multivariate approach to quantify how physical figures of merit related to image quality change with acquisition/reconstruction/patient-dependent parameters in a phantom experiment.

Methods: The NEMA-IQ phantom was used to evaluate contrast recovery coefficient (CRC), background variability (BV) and contrast-to-noise ratio (CNR) as a function of changing emission scan duration (ESD), activity concentration (AC), target internal diameter (ID), target-background activity ratio (TBR), and body mass index (BMI). The phantom was filled with an average concentration of $5.3 \mathrm{kBq} / \mathrm{mL}$ of FDG solution and the spheres with TBR of 21.2, 8.8, and 5.0 in 3 different sessions. Images were acquired at varying background activity concentration from 5.1 to 1.3 $\mathrm{kBq} / \mathrm{mL}$ and images were reconstructed for ESD of 30-151 seconds per bed position with and without Point Spread Function (PSF) correction. The parameters were all considered in a single analysis using multiple linear regression methods.

Results: As expected, CRC depended only on sphere ID and on PSF application, while BV depended on sphere ID, ESD, AC and BMI of the phantom, in order of decreasing relevance. Noteworthy, ESD and $\mathrm{AC}$ resulted as the most significant predictors of CNR variability with a similar relevance, followed by the weight of the patient and TBR of the lesion.

Conclusions: AC and ESD proved to be effective tools in modulating CNR. ESD could be increased rather than AC to improve image quality in overweight/obese patients to fulfil ALARA principles. 
1 Keywords: Positron emission tomography, time-of flight, 18FDG, Contrast-to-noise ratio, optimization, image quality

3

4

5 


\section{Background}

2 Thanks to the improvements in hardware components and in imaging reconstruction techniques, significant advances have been made in recent years in positron emission tomography / computed tomography (PET/CT) systems [1,2]. They are mainly related to the use of fast detectors, Lutetium Oxyorthosilicate (LSO) and/or Lutetium Yttrium Oxyorthosilicate (LYSO) coupled to both Time-ofFlight (TOF) technology and advanced reconstruction modalities such as the modelling of the system Point-Spread-Function (PSF) $[3,4,5]$ and/or noise [6] which improve the accuracy of quantitative information and enhance the detectability of small lesions $[7,8]$. The main motivation for TOF-PET has always been the potential image quality improvement or reduction in image acquisition time [1,2]. The effective sensitivity gain was already described nearly 40 years ago $[8,9]$ as depending on the ratio between the object size $D$ and the spatial FWHM of the TOF kernel $\Delta x$.

In oncology practice, typically a longer acquisition time is needed for a larger patient characterized by higher attenuation. Often the longer acquisition time does not compensate for the poor quality of 
1 However, precise information on how to tune administered FDG activity and emission scan duration

2 in whole-body oncological studies on TOF-PET/CT scanners, is still a demanding need for the nuclear

3 medicine physicians.

4 Few papers in the literature studied the optimization of ${ }^{18}$ FDG activity administration $[17,18]$ or

5 emission scan duration [19] on TOF-PET/CT scanners, but they examined the two factors

6 independently.

7 The aim of this work was to describe how the physical figures of merit related to PET image quality

8 change with different acquisition, reconstruction and object dependent parameters on a TOF-PET/CT

9 scanner. The study was designed to simultaneously analyse the impact of the different factors with a multivariable approach, using phantoms with a variable weight, which hosted several well-defined target sizes with a known target-to -background ratio, as done in previous study [20]. We selected the emission scan duration (ESD), the FDG activity concentration (AC), the target-to-background activity concentration ratio (TBR), the target size (ID), the body mass index (BMI) of scanned object and the application of PSF correction (PSF) as the factors that could affect the Contrast Recovery Coefficient (CRC), the Background Variability (BV) and the Contrast-to-Noise Ratio (CNR), identified as PET image quality descriptors.

\section{Methods}

\section{$\underline{\text { PET/CT scanner }}$}

The Ingenuity TF 64 (Philips Healthcare, Cleveland, OH, USA) is a Lutetium-Yttrium Oxyorthosilicate (LYSO) TOF PET/CT scanner. The energy window is set between 440 and $665 \mathrm{keV}$ and the coincidence window is $3.8 \mathrm{~ns}$.

The performance characteristics of this TOF-PET/CT scanner according to NEMA NU 2012 standard have already been described in detail [21]. 
Phantom setup

2 The International Electrotechnical Commission (IEC) 61675-1 emission phantom (NEMA image quality phantom, NEMA-IQ phantom) with ${ }^{18} \mathrm{~F}$ solution was used. The NEMA-IQ phantom has an interior cavity volume of $9947 \mathrm{~mL}$ and contains 6 fillable spheres with 10, 13, 17, 22, 28, and $37 \mathrm{~mm}$

5 inner diameters (ID). A cylindrical insert filled with low density foam (density of $0.30 \mathrm{~g} / \mathrm{cm}^{3}$ ) was 6 fixed along the centre of the phantom. Four micro-hollow spheres with ID of 4.1, 4.7, 6.5, and 8.1 $7 \mathrm{~mm}$ (Data Spectrum Corporation) were fixed to a foam support attached to the lung insert at the bottom of the phantom, as shown in Figure 1a. The microspheres are tightly fixed to the foam support,

9 in a unique position that guarantees the microspheres to be set always at the same height with respect to the base of the phantom to exclude possible bias in the further analysis.

The NEMA-IQ phantom was centred in the transverse FOV of the scanner with the equatorial plane of the standard spheres coplanar to the centre of the axial FOV.

To simulate the activity outside the scanner FOV, the scatter phantom (Data Spectrum Corporation) was placed close at the end of the IEC phantom, strictly following NEMA NU-2 recommendations [22]. It is a solid circular cylinder composed of polyethylene with outside diameter of $203 \mathrm{~mm}$ and a length of $700 \mathrm{~mm}$. A $6.4 \mathrm{~mm}$ hole is drilled along central axis of the cylinder. A $700 \mathrm{~mm}$ polyethylene tube with an inside diameter of $3.2 \mathrm{~mm}$ and an outside diameter of $4.8 \mathrm{~mm}$ is placed in the hole.

Finally, to simulate a different patient habitus, a 'belt' of 11 water bags of $500 \mathrm{~mL}$ and $3 \mathrm{~cm}$ thick was fit over the NEMA-IQ phantom (Figure 1b) [20, 23]. A few papers in the literature [24, 25] developed methods to assess the Size Specific Dose Estimate in patients undergoing chest and abdomen CT examinations, based on the relation between the body mass index (BMI) and the effective diameter $d_{\text {eff }}$ of the patient:

$$
\mathrm{d}_{\mathrm{eff}}(\mathrm{cm})=0.6414\left(\mathrm{~cm} \cdot \mathrm{m}^{2} / \mathrm{kg}\right) \times \mathrm{BMI}+12.976(\mathrm{~cm})
$$


BMI was calculated accordingly to the formula provided by the World Health Organization [26], $\mathrm{BMI}=\left(\right.$ weight $\mathrm{x}$ height $\left.^{-2}\right)$ and $\mathrm{d}_{\mathrm{eff}}=\sqrt{\mathrm{d}_{\mathrm{LL}} \mathrm{x}} \mathrm{d}_{\mathrm{AP}}$, being $\mathrm{d}_{\mathrm{LL}}, \mathrm{d}_{\mathrm{AP}}$ the latero-lateral and antero-posterior diameter of the patient.

From the measure of the $\mathrm{d}_{\mathrm{AP}}$ and $\mathrm{d}_{\mathrm{LL}}$ made on the CT scan of both NEMA-IQ and NEMA-IQ fitted with the additional belt, BMI values were calculated by means of the relation above for the two configurations.

\section{Phantoms preparation and acquisition}

The spheres of the NEMA-IQ phantom were filled with ${ }^{18} \mathrm{~F}$ activity concentrations of $117.3,46.6$ and $25.6 \mathrm{kBq} / \mathrm{ml}$ and the IEC cavity with ${ }^{18} \mathrm{~F}$ activity concentration of $5.5,5.3$ and $5.1 \mathrm{kBq} / \mathrm{ml}$, in three different experimental sessions, respectively, thus providing 21.2, 8.8 and 5.0 target-to-background ratios (TBR). The reference time for all the activity concentrations is the time of the first acquisition, as described in the paragraph below.

A more clinical parameter used to evaluate the uptake in a lesion is the Standardize Uptake Value (SUV) which describes the activity concentration in the lesion with respect to the total activity concentration in the phantom (this last being normally higher than the background concentration). The correspondent theoretical SUV values realized in the three experimental sessions were 19, 8 and 5 , respectively.

The capillary present in the scatter phantom was filled with ${ }^{18} \mathrm{~F}$ activity concentrations of 5.3, 5.1, 5.3 $\mathrm{kBq} / \mathrm{ml}$ in the three experimental sessions, respectively.

PET/CT images of both NEMA-IQ and NEMA-IQ wrapped with the belt (b-NEMA-IQ) were acquired in list mode with the NEMA NU-2 2012 protocol with one bed of $151 \mathrm{sec}$ to simulate a total body scan of $100 \mathrm{~cm}$ axial imaging distance in 30 minutes of emission imaging and in accordance to the manufacturer's protocol [27]. NEMA-IQ and b-NEMA-IQ phantoms were acquired sequentially, at different activity concentrations of about 5.1, 3.1, 2.2, 1.5 and $1.3 \mathrm{kBq} / \mathrm{ml}$. Overall 30 (5 activity 
concentrations x two phantoms x 3 sessions) acquisitions were performed. A CT scan was used for attenuation correction.

(1)

\section{Image reconstruction}

After correction for attenuation, scatter, random, detector normalization, radioactive decay, system dead time and crystal timing, images were reconstructed using a TOF, list-mode, blob-based, ordered subsets maximum likelihood expectation maximization algorithm (TOF-OSEM) [28].

The standard protocol provided by the manufacturer for clinical whole-body examinations was used to reconstruct all the images acquired, by setting the Speed to Normal (99 equivalent iterations and a TOF kernel width of $14.1 \mathrm{~cm}$ ), the Smooth to Normal (full width at half maximum FWHM of the Gaussian filter equal to $4 \mathrm{~mm}$ and the relaxation parameter equal to 1.0) on a $144 \mathrm{x} 144$ frame (4 mm isotropic voxel). The reconstructions were performed for different emission scan durations (ESD) with $(\mathrm{PSF}=1)$ and without $(\mathrm{PSF}=0)$ the application of the resolution recovery algorithm or PSF correction (PSF) as shown in Table 1. On the Ingenuity TF PET/CT scanner, PSF correction is characterized by two parameters, the number of iterations and the regularization kernel width (FWHM expressed in $\mathrm{mm}$ ). The default parameters suggested by the manufacturer (iteration $=1$, regularization $=6 \mathrm{~mm}$ ) were used when PSF was applied.

The different ESD were obtained by cutting the list-mode file after ESD seconds. Namely, ESD was set to $30,45,60,75,90$ and 120 seconds. The PSF, speed and smoothing filter parameters were kept fixed for each reconstruction, as no significant difference in contrast recovery coefficient and background variability exists by changing speed, smooth and PSF values [21].

Table 1 Reconstruction parameters values used in phantom image reconstruction 


\section{Reconstruction parameter $\quad$ Values}

PSF correction (on/off) $\quad 1$ iteration, $6 \mathrm{~mm}$ regularization

Speed Normal

3 iterations, 33 subsets

Smooth Normal

4 mm Gaussian filter FWHM

$\underline{\text { Image analysis }}$

The percentage Contrast Recovery Coefficient (CRC) and the Background Variability (BV) were evaluated by a routine provided by the manufacturer, according to NEMA NU-2 2012 standards:

$$
\begin{aligned}
& C R C=\frac{\left(\frac{C_{S j}}{C_{B j}}\right)-1}{\left(\frac{A_{S}}{A_{B}}\right)-1} \times 100 \% \\
& B V_{j}=\frac{S D_{j}}{C_{B j}} \times 100 \%
\end{aligned}
$$

where: $S D_{j}=\sqrt{\sum_{1}^{k}\left(C_{B j k}-C_{B j}\right)^{2} /(k-1)}$

$$
\mathrm{C}_{\mathrm{Sj}}=\text { average counts in the ROI for sphere } \mathrm{j}
$$$$
\mathrm{C}_{\mathrm{Bj}}=\text { average of the background counts for sphere } \mathrm{j}
$$

$\mathrm{A}_{\mathrm{S}}=$ activity concentration in the spheres

$A_{B}=$ activity concentration in the background

This routine provides the user with a pattern of six ROIs of fixed diameters equal to the physical internal diameter of the spheres and fixed relative distances. After choosing the slice corresponding to the equatorial section of the spheres, the user can only rotate and translate the pattern to establish its correct position over the hot spheres in the central slice. A pattern of twelve groups of $37 \mathrm{~mm}$ background ROIs at a distance of $15 \mathrm{~mm}$ from the edge of the phantom but no closer than $15 \mathrm{~mm}$ to any sphere is linked to the pattern of the six ROIs and is automatically placed in the background. The positioning of the smaller ROIs $(10,13,17,22$, and $28 \mathrm{~mm})$ on background, concentric to the $37 \mathrm{~mm}$ ones, is done automatically. The same pattern of 12 background ROIs is automatically positioned at 
1 a distance of \pm 1 and $\pm 2 \mathrm{~cm}$ from the central slice for a total of $K=60$ background ROIs, as prescribed by NEMA recommendations. The analysis of CRC and BV for the four microspheres was performed by means of a routine developed on purpose in ImageJ v 1.48 (National Institutes of Health, Bethesda, Maryland, USA [29]). The ImageJ routine was written to act similarly to the manufacturer's one, with the difference that the pattern provided contained 4 ROIs of fixed diameters equal to the physical internal diameter of the microspheres and fixed relative distances correspondent to their positions. The background ROIs pattern consisted of 12 groups of concentric ROIs of 4.1, 4.7, 6.5 and $8.1 \mathrm{~mm}$ diameters.

Both the routines output $\mathrm{CRC}$ and $\mathrm{BV}$ values for each sphere and $\mathrm{ROI}$ dimension, as well as the mean and standard deviation of the activity concentration for all the ROIs drawn.

Moreover, the contrast-to-noise ratio (CNR), which is the physical figure of merit more closely related to lesion detectability, was evaluated for all the spheres that were detected on the reconstructed images. In accordance with [18], CNR for sphere $\mathrm{j}$ was defined as: two nuclear medicine physicians using a five-point rating scale from 0 to 4 to answer the question "Is the sphere visible?" The scores were defined as: $0=$ definitely no; $1=$ probably no; $2=$ possibly yes;

$$
C N R j=\frac{\left(C_{S j}-C_{B}\right) / C_{S j}}{S D_{B} / C_{B}}
$$

where: $\mathrm{C}_{\mathrm{Sj}}=$ average counts in the ROI for sphere $\mathrm{j}$

$C_{B}=\frac{1}{J} \sum_{1}^{J} C_{B j}, C_{\mathrm{Bj}}$ is the average counts measured in all background ROIs of size $\mathrm{j}$, $S D_{B}=\sqrt{\sum_{1}^{J} \sum_{1}^{K}\left(C_{B j, k}-C_{B}\right)^{2} /(K \cdot J-1)}, \mathrm{J}=8$ (accounting for the number of visible spheres) and $\mathrm{K}=12$ (accounting for the ROI positions in the phantom background).

Overall, 3360 CNR values were evaluated (8 visible ID x 7 ESD x 3 TBR x 5 Aacq x 2 phantoms x 2 PSF values).

Finally, the visibility of the hot spheres in the reconstructed images was assessed independently by $3=$ probably yes; 4=definitely yes. Three sets of consecutive transaxial image planes were displayed 
1 for each case to be rated, allowing the reader to evaluate the central plane in the context of adjacent

2 planes. Readers were able to adjust the lookup table of each image to facilitate image viewing. The order in which images sets were evaluated was randomized.

\section{$\underline{\text { Statistical Analysis }}$}

Correlation matrices were used to identify potential univariate correlations between image quality figures of merit (CRC, BV and CNR) and acquisition (ESD, AC), reconstruction (PSF application) and object dependent parameters (ID, TBR and BMI). Only significant predictors at univariate analysis were considered and inserted into multiple linear regression methods to derive analytical formulas of the model.

The weight of different independent variables in explaining the dependent variables were quantified by means of standardized regression coefficients $(\beta)$. The $\beta$ s can be used as a measure of relative importance, with the independent variables ranked in order of the sizes of these coefficients (ignoring sign) [30].

Box and whiskers plots were used to provide a univariate graphical representation of CRC and BV with respect to significant predictors, identified by the regression models.

The impact of the different acquisition and object dependent parameters on CNR, was further investigated by a multiple way principal effects ANOVA: acquisition and object dependent parameters were considered as independent variables (factors) and CNR as the dependent variables A post-hoc test (Scheffe' $F$ test) was performed to identify the main sources of variability. If a significant $\mathrm{F}$ value was found for one independent variable, then this was referred as a main effect. When a main effect was found, then the Scheffe' test was performed to compare the dependent variable upon the levels of the factor $2 \times 2$, thus identifying the main sources of variability. These comparisons were represented by drawing the least squares means, which are the best linear estimates for the marginal means in the ANOVA design, together with the standard errors of the means (and 
thus the $95 \%$ confidence intervals) [30]. The reproducibility of visual scoring of hot spheres in the reconstructed images was estimated by the correlation agreement using Cohen weighted Kappa (kw). All statistical analyses were performed with the software STATISTICA 6.0 (Statsoft Inc, USA).

Results

\section{$\underline{\text { Phantoms }}$}

The $d_{A P}$ and $d_{L L}$ measured on the CT scan were 22.9 and $29.5 \mathrm{~cm}$ and 27.9 and $36.1 \mathrm{~cm}$ for the NEMA-IQ and the b-NEMA-IQ phantoms, respectively, resulting in effective diameters of 26.0 and $31.7 \mathrm{~cm}$, respectively (Figure $1 \mathrm{~b}$ ). By using the relation (1), the $\mathrm{d}_{\mathrm{eff}}$ values corresponded to BMI values of 21.4 and $29.2 \mathrm{~kg} / \mathrm{m}^{2}$ for NEMA-IQ and b-NEMA-IQ, respectively and allow to classify the two phantoms as simulating normal and high-overweight patients.

\section{$\underline{\text { Contrast recovery coefficient }}$}

The recovery of ${ }^{18} \mathrm{~F}$ activity in the spheres of NEMA-IQ phantom depends on sphere ID $\left(\square_{\mathrm{ID}}=0.68\right)$ and on the application of PSF correction $\left(\square_{\mathrm{PSF}}=0.23\right)$, in order of decreasing relevance of the weight of the variable in the model. F was $1382(\mathrm{p}<0.0001)$. The adjusted $\mathrm{R}^{2}$ of model fitting was 0.51 and the residual were normally distributed.

Figure 2 shows box plot of CRC with respect to sphere ID (a) and PSF correction (b).

\section{$\underline{\text { Background variability }}$}

The multiple linear regression analysis showed that BV depends on sphereID $(\square \mathrm{ID}=-0.65)$, ESD $(\square \mathrm{ESD}=-0.40), \mathrm{AC}\left(\square_{\mathrm{AC}}=-0.35\right)$ and $\mathrm{W}\left(\square_{\mathrm{w}}=0.25\right)$, in order of decreasing relevance. $\mathrm{F}$ was 1641 ( $\mathrm{p}<0.0001)$. The adjusted $\mathrm{R}^{2}$ of model fitting equals to 0.71 and the residual were normally distributed. The multiple linear regression equation that summarizes the model with BV as predicted variable and sphere ID, ESD, AC and BMI as predictors can be written as: 
$2 \quad \mathrm{BV}=23.7-\left(0.5 m m^{-1} \times\right.$ sphereID $)-\left(0.1 \mathrm{~s}^{-1} \times \mathrm{ESD}\right)-\left(1.8 \frac{\mathrm{ml}}{\mathrm{kBq}} \times \mathrm{AC}\right)+\left(0.4 \frac{\mathrm{m}^{2}}{\mathrm{~kg}} \times \mathrm{BMI}\right)$

3

Figure 3 shows box plot of BV with respect to sphere ID (a), ESD (b), AC (c) and BMI (d), respectively.

\section{$\underline{\text { Contrast to noise ratio }}$}

The CNR of the spheres detected on the images depends on $\mathrm{ESD}\left(\square_{\mathrm{ESD}}=0.53\right), \mathrm{AC}\left(\square_{\mathrm{AC}}=0.50\right), \mathrm{BMI}$ $(\square \mathrm{w}=-0.37)$ and TBR $(\square \mathrm{ESD}=0.26)$, in order of decreasing relevance. $\mathrm{F}$ was $1528(\mathrm{p}<0.0001)$. The adjusted $\mathrm{R}^{2}$ of model fitting equals to 0.73 and the residuals were normally distributed. The regression equation that best summarizes the results obtained in a multiple regression model for CNR is:

$\mathrm{CNR}=4.87+\left(0.03 s^{-1} \times \mathrm{ESD}\right)+\left(0.74 \frac{\mathrm{ml}}{k B q} \times \mathrm{AC}\right)-\left(0.19 \frac{\mathrm{m}^{2}}{\mathrm{~kg}} \times \mathrm{BMI}\right)+(0.08 \times \mathrm{TBR})$

ESD and AC impact with a similar weight on CNR. As expected, BMI impacts with a negative regression coefficient on CNR, i.e. as the BMI increases the CNR decreases. Only last came TBR, with a slight impact on CNR about one half the one of ESD and AC. The major gain in CNR was observed for low TBR (or low SUV) values, as when moving from TBR 5.0 to 8.8 the CNR increases of about $19 \%$, while when moving from TBR 8.8 to 21.2 the CNR increase is only of about $9 \%$ (Figure 4d). However, this is an intrinsic characteristic of the lesion itself and cannot be managed in the optimization process.

Post-hoc Scheffè test showed a statistically significant increase in CNR for every contrast between adjacent levels of $\mathrm{AC}$ in the range explored $(\mathrm{p}<0.001)$ (Figure $4 \mathrm{a})$. When considering $2.2 \mathrm{kBq} / \mathrm{mL}$, which represents the activity concentration 60 minutes post injection of $3 \mathrm{MBq} / \mathrm{kg}$ of $18 \mathrm{~F}-\mathrm{FDG}$, the 
1 CNR mean value increases of about $19 \%$ when moving to an activity concentration of $3.1 \mathrm{kBq} / \mathrm{mL}$

2 (which correspond to an injection scheme of $4.5 \mathrm{MBq} / \mathrm{kg}$ ).

3 A similar behaviour was observed for all the ESD, BMI and TBR contrast tested. Post-hoc Scheffè test showed a statistically significant increase in CNR for every contrast between adjacent levels of ESD (Figure 4b), BMI (Figure 4c) and TBR (Figure 4d) in the range explored $(\mathrm{p}<0.001)$.

\section{$\underline{\text { Visual detection of the spheres }}$}

Interobserver reproducibility analysis showed an excellent reproducibility between the two observers $\left(\mathrm{k}_{\mathrm{W}}=0.87\right)$. A hot sphere was considered visually detectable if its median score was 2 . Figure 5a and $5 b$ show the results of visual detection of different sphere ID for different TBR, AC and ESD for NEMA-IQ and b-NEMA-IQ phantoms, respectively. From these data it is evident that the overall scores for the lesions in the NEMA-IQ phantom are higher than those for the lesions in the b-NEMAIQ one, confirming the impact of the BMI on lesion detectability.

In particular, one can observe that this PET/CT scanner is unlikely to detect lesions with dimension of $6.5 \mathrm{~mm}$ or less with TBR 21.2 or lower, lesions of dimension $8,1 \mathrm{~mm}$ or less with TBR is 8.8 or lower, and lesions of dimension of $13 \mathrm{~mm}$ or less with TBR ratio is 5.0 or lower. Moreover, the increase in activity concentration and emission scan duration appears to be particularly important to achieve lesion detection for the b-NEMA-IQ phantom configuration.

Figures $6 \mathrm{a}$ and $6 \mathrm{~b}$ show NEMA-IQ and b-NEMA-IQ reconstructed images obtained for TBR $=8.8$ with increasing of $\mathrm{AC}(\mathrm{ESD}=60 \mathrm{~s})$ and $\mathrm{ESD}(\mathrm{AC}=2.0 \mathrm{kBq} / \mathrm{ml})$, respectively.

\section{Discussion}

The optimization process invoked by the Euratom Directive 2016-59 [31], requires optimal image quality of FDG whole-body PET/CT examinations by opportunely tuning acquisition and reconstruction parameters as well as the FDG activity to administer to patients. This process must be 
performed in the light of reducing the radiation dose burden in particular to patients frequently exposed to several radiological examination during their follow up.The image quality and lesion detectability in ${ }^{18}$ F-FDG PET imaging are limited by different factors as the low signal-to-noise ratio, the relatively low spatial resolution and patient's motion, which results in the partial-volume effect affecting lesion visualization and quantitation [2].

This work was aimed to characterize the quality of PET images for a TOF-PET/CT scanner in a wide range of acquisition, reconstruction and object dependent parameters in settings like those encountered in clinical practice, by means of a phantom study. CRC, BV and CNR, which is closely related to lesion detectability, were the figures of merit used to describe PET image quality. Our study used a multivariate approach to quantify how these figures of merit change as a function of ESD and AC for different target size, TBR, BMI and under the effect of the point spread function correction.

The main result of this study is that the CNR of FDG lesions depends on ESD and AC in a similar way $(\square=0.53$ and $\square=0.50)$. This result is not obvious, if one considers that different FDG administration schemes are proposed by the literature. A previous study performed on a non-TOF PET scanner [20] concluded that the main predictor of CNR was ESD $(\square=0.60)$ and only with a half of the explanatory power $(\square=0.27)$ came AC. On the other hand, EANM guidelines [13] provides administration schemes based on a linear and a quadratic relationship [15], respectively, between PET acquisition time per bed position, patient weight and recommended FDG activity. Moreover, the difference in CNR dependence on ESD and AC on the two PET/CT scanners may rely on the TOF technology that is available in the PET/CT scanner used in the present study, but not in [20]. As the reduction of acquisition time is one the main achievement of TOF technology, this last can be the reason for the reduced dependence of CNR on ESD with respect to non-TOF PET scanners. Moreover, our results show also a significant increase in CNR for each increasing step in AC or ESD in the range explored, i.e. from 1.3 to $5.1 \mathrm{kBq} / \mathrm{ml}$ and from 30 to $151 \mathrm{sec}$. The visual inspection of phantom images confirmed that the image quality, in terms of the noise level and contrast, can be improved by increasing the AC (Figure 4a) or ESD (Figure 4b). This finding agrees with the clinical 
results reported in the recent paper of Prieto [18]. The author observed a statistically significant difference in both the image noise and the overall image quality indexes of PET images obtained after 18FDG activity administration of 5.2 and $3.7 \mathrm{MBq} / \mathrm{kg}$ on the Siemens mCT TOF-PET/CT scanner. The third predictor of CNR was the BMI of the phantom $(\square=-0.37)$, indicating that on average the CNR decreased by $25 \%$ with increasing the BMI of the scanned object by a factor of 1.4 . It is known that the TOF technology allowing the reduction of the uncertainty on the annihilation event acts as a noise equalizer and brings an overall gain in signal-to noise ratio, being this effect more evident for larger object $[1,10]$. However, notwithstanding TOF technology, this result confirms that there is still a residual dependence of CNR on the size of the imaged object as evidenced by the literature [1]. The b-NEMA-IQ phantom realized in this study, with a BMI of $29.6 \mathrm{~kg} / \mathrm{m}^{2}$ calculated from its $\mathrm{d}_{\text {eff }}$, simulates a high-overweight patient. A way to improve lesion detection is then to define specific acquisition protocols for oncological whole-body studies tailored on patient's BMI, rather than using a fixed ESD. Should this result be confirmed in clinical studies, it would indicate an additional way to improve the lesion detectability for larger patients, depending on their weight or their body habitus. The last predictor of CNR was the TBR of the lesion $(\square=0.26)$. This dependence may be explained by the partial volume effect, which reduces the apparent activity concentration in the lesion in the reconstructed image preventing the recovering of the true amount of activity for structures less than twice the reconstructed image resolution.

The fitted multiple regression model of CNR based on these premises, accounts for more than two thirds of CNR variance (adjusted $\mathrm{R}^{2}=0.76$ ).

From these findings, one can derive that it is possible to opportunely tune ESD and AC on patient's BMI in order to keep constant the CNR, or the detectability level, on the PET images of this scanner. As a typical example, let us consider the following situation: a lesion with a TBR of 9 (SUV=8.8) in a standard BMI patient injected with $3 \mathrm{MBq} / \mathrm{kg}$ of ${ }^{18} \mathrm{~F}-\mathrm{FDG}$ imaged for $60 \mathrm{sec}, 60$ minutes post injection. According to equation (4), we should expect a CNR of 5.9 for a $10 \mathrm{~mm}$-diameter lesion. To obtain a similar value in CNR for the same lesion uptake in a high overweight patient injected with 3 
$\mathrm{MBq} / \mathrm{kg}$ of $18 \mathrm{~F}-\mathrm{FDG}$, the patient should be scanned for 110s. In an analogous way, one could double the activity administration scheme (i.e. $6 \mathrm{MBq} / \mathrm{kg}$ ) to obtain the same CNR. However, from a radiation protection point of view, it would be more advisable to increase the ESD than the AC for an improvement in lesion detectability, still considering that a long ESD would increase the risk of patient's movement.

Another result of this study is the dependence of CRC on sphere ID $(\square=0.68)$ and PSF application $(\square=0.23)$. This result confirms the dependence of CRC already reported by Zorz et al. [21], even with a slightly different analytical expression with respect to (2). This may be explained by observing that in [21] only four spheres (ID = 10,13,17 and 22mm) were included in NEMA-IQ phantom analysis and CRC fitting was almost perfectly linear $\left(\mathrm{R}_{\mathrm{adj}}{ }^{2}=0.93\right)$. In our study, on the contrary, CRC dependence on ID was analysed in a wider sphere dimension range, where CRC values assume a trend with respect to ID (Figure 1a) definitely different from the linear one, explaining also the relatively low $\mathrm{R}_{\mathrm{adj}}{ }^{2}$ of 0.51 .

The third parameter related to image quality we investigated, the BV resulted to be dependent on the ROI dimension for which is defined $(\square=-0.62)$, emission scan duration $(\square=-0.39)$, activity concentration $(\square=-0.31)$ and BMI $(\square=0.24)$, in order of decreasing relevance, this model explaining $77 \%$ of variance in $\mathrm{BV}\left(\mathrm{R}_{\mathrm{adj}}{ }^{2}\right.$ of 0.77$)$. The strong dependence of $\mathrm{BV}$ on sphere ID is not new and was also found by Zorz [21] and Brambilla [20], with similar weights. Even if BV cannot be considered a descriptor of noise, it is interesting to note the equivalent dependence of BV on ESD ( $\beta$ $=-0.39)$ and $\mathrm{AC}(\beta=-0.31)$, in analogy with the finding that CNR depends on both these parameters with similar weights, reported above. The impact of the BMI of the phantom on BV, reinforces the same finding on CNR.

Some limitations of the present study must be acknowledged.

First, the results on CNR, CRC and BV found in the present study strictly apply to this PET/CT scanner and to $18 \mathrm{~F}$ whole body acquisitions: extrapolation of these results to different TOF-PET/CT scanners and to different radionuclides should be tested in advance before application. 
1 Second, the 'belt' added to the NEMA-IQ phantom to simulate a high overweight patient was filled

9

\section{.} 1 2 3 with non-radioactive water, while in obese patients, the adipose tissue is mildly radioactive, typical SUVs being around $0.3 \mathrm{~g} / \mathrm{ml}$. When considering the_attenuation and the scatter caused by the belt on the photons coming from the NEMA-IQ phantom, these effects would be equivalent to those originating from a $18 \mathrm{~F}$ filled belt. Nevertheless, the photons emitted from the radioactive belt will cause an increase in the noise and thus a deterioration of the quality of the NEMA-IQ phantom image. Another issue concerning the 'belt' is the presence of air gaps between the water bags and the NEMAIEC phantom, as Figure 1 shows, the air gaps accounting for less than the $10 \%$ of the total area of section of the b-NEMA-IEC phantom, reducing the attenuation and the scatter phenomena effects. In ultimate analysis, both the non-radioactive water and the presence of air gap between the 'belt' and the NEMA-IQ phantom are in some way an ameliorative condition of the real clinical situation. Thus, in this scenario, the conclusion that the image quality in overweight patients is worsened, is reinforced.

Third, the NEMA-IQ and the b-NEMA-IQ phantoms represent the thorax-abdominal regions of a patient. For a comprehensive analysis of the effect of activity concentration, emission scan duration and target-to-background ratio on image quality, the simulation of head and neck and pelvis regions should be devised. To this end, clinical studies would help in confirming the results of the present study.

\section{Abbreviations}

5

\footnotetext{
TOF: Time-of-flight
} 
1 FDG: ${ }^{18}$ F-fluoro-2-deoxyglucose

2 PET: Positron Emission Tomography

3 CT: Computed Tomography

4 CRC: Contrast Recovery Coefficient

$5 \quad$ BV: Background Variability

6 CNR: Contrast to Noise Ratio

7 ESD: Emission Scan Duration

8 AC: Activity Concentration

9 ALARA: As Low As Reasonably Achievable

10 ID: Internal Diameter

11 TBR: Target-Background activity Ratio

12 BMI: Body Mass Index

13 LSO: Lutetium Oxyorthosilicate

14 LYSO: Lutetium Yttrium Oxyorthosilicate

15 PSF: Point Spread Function

16 FWHM: Full Width at Half Maximum

17 NEMA: National Electrical Manufacturer Association

18 IEC: International Electrotechnical Commission

19 b-NEMA-IQ: NEMA-IQ phantom wrapped with belt

20 OSEM: Ordered Subset Expectation Maximization 
Declarations

Ethics approval and consent to participate

Not applicable

\section{Consent for publication}

Not applicable

\section{Availability of data and material}

The datasets used and analyzed during the current study are available from the corresponding author on reasonable request.

\section{Competing interests}

The authors declare that they have no competing interests.

\section{Funding}

Not applicable

\section{Authors' contributions}

$\mathrm{RM}, \mathrm{MB}$ and AC contributed to the design of the study. RM, NAM and AO performed the data acquisition with the support from GMS. NAM and AO reconstructed and analysed the images. RM drafted this paper, which was revised by MB and GMS. All authors approved the final manuscript.

\section{Acknowledgements}

Not applicable 
1 Vandenberghe S, Mikhaylova E, D'Hoe E, Mollet P, Karp JS Recent developments in time-of-flight PET. EJNMMI Phys. 2016 Dec;3(1):3. doi: 10.1186/s40658-016-0138-3

2 Van der Vos CS, Koopman D, Rijnsdorp S et al. Quantification, improvement, and harmonization of small lesion detection with state-of-the-art PET. Eur J Med Mol Imaging 2017; 44(Suppl 1): S4S16.

3 Lecomte R, Schmitt D, Lamoureux G. Geometry study of a high resolution PET detection system using small detectors. IEEE Trans Nucl Sci 1984;31:556-61

4 Alessio AM, Kinahan PE, Lewellen TK. Modeling and incorporation of system response functions in 3-D whole body PET. IEEE Trans Med Imaging 2006;25: 828-37.

5 Panin VY, Kehren F, Michel C, Casey M. Fully 3-D PET reconstruction with system matrix derived from point source measurements. IEEE Trans Med Imaging 2006;25:907-21.

6 Teoh EJ, McGowan DR, Macpherson RE, Bradley KM, Gleeson FV. Phantom and clinical evaluation of the Bayesian penalized likelihood reconstruction algorithm Q.Clear on an LYSO PET/CT system. J Nucl Med 2015;56(9):1447-52. doi:10.2967/jnumed.115.159301

7 Rahmim A, Tang J. Noise propagation in resolution modeled PET imaging and its impact on detectability. Phys Med Biol 2013;58:6945-68.

8 Knäusl B, Rausch IF, Bergmann H, Dudczak R, Hirtl A, Georg D. Influence of PET reconstruction parameters on the TrueX algorithm. A combined phantom and patient study. Nuklearmedizin 2013;52: 28-35.

9 Budinger TF Time-of-flight positron emission tomography: status relative to conventional PET. J Nucl Med. 1983;24:73-8

10 Conti M Focus on time-of-flight PET: the benefits of improved time resolution, Eur J Nucl Med Mol Imaging (2011) 38:1147-1157. DOI 10.1007/s00259-010-1711-y

11 Karp JS, Surti S, Daube-Witherspoon ME, Muehllehner G. Benefit of Time-of-Flight in PET: experimental and clinical results. J Nucl Med 2008;49:462-470

12 Lois C, Jakoby BW, Long MJ et al. An assessment of the impact of incorporating Time-of-Flight information into clinical PET/CT imaging. J Nucl Med 2010;51:237-245.

13 Boellaard R, Delgado-Bolton R, Oyen WJG, Giammarile F, Tatsch K, Eschner W, et al. FDG PET/CT: EANM procedure guidelines for tumour imaging: version 2.0. Eur J Nucl Med Mol Imaging 2015;42:328-54. 
14 Boellaard R,Willemsen AT, Arends B, Visser EP. EARL procedure for assessing PET/CTsystem specific patient FDG activity preparations for quantitative FDG PET/CT studies.

http://earl.eanm.org/html/img/pool/EARL-procedure-for-optimizing-FDG-activity-for-quantitativeFDGPET-studies_version_1_1.pdf. Accessed online 30 June 2020.

15 de Groot EH, Post N, Boellard R, Wagenaar NRL, Willemsen TM and van Dalen JA. Optimized dose regimen for whole body FDG-PET imaging. EJNMMI Research 2013;63. http://www.ejnmmires.com/content/3/1/63

16 Karakatsanis NA, Fokou E and Tsoumpas Charalampos. Dosage optimization in positron emission tomography: state-of-the-art methods and future prospects. Am J Nucl Med Mol Imaging 2015;5(5):527-547.

17 Schaefferkoetter J, Nai YH, Reilhac A et al. Low dose positron emission tomography emulation from decimated high statistics: A clinical validation study. Med. Phys. 2019;46:26382645.https://doi.org/10.1002/mp.13517

18 Prieto E, Garcia-Velloso MJ, Rodriguez-Fraile M, et al. Significant dose reduction is feasible in FDG PET/CT protocols without compromising diagnostic quality. Phys Med. 2018 Feb;46:134-139. doi: 10.1016/j.ejmp.2018.01.021.

19 Murray I, Time-of-flight PET/CT using low-activity protocols: potential implications for cancer therapy monitoring, Eur J Nucl Med Mol Imaging (2010) 37:1643-1653 DOI 10.1007/s00259-0101466-5.

20 Brambilla M, Matheoud R, Secco C, et al. Impact of target-to-background ratio, target size, emission scan duration, and activity on physical figures of merit for a 3D LSO-based whole body PET/CT scanner. Med Phys. 2007;34:3854-65.

21 Zorz A, Matheoud R, Richetta E, Baichoo S, Poli M, Scaggion A et al. Performance evaluation of a new time of flight PET/CT scanner: Results of a multicenter study. Phys Med 2019;68:146-154. doi: 10.1016/j.ejmp.2019.11.017.

22 NEMA NU 2-2012 Performance Measurements of Positron Emission Tomographs. National Electrical Manufacturers Association, Rosslyn, VA 22209.

23 Grosser OS, Kupitz D, Ruf J, Czuczwara D, Steffen IG, Furth C et al. Optimization os SPECTCT hybrid imaging using iterative image reconstruction for low dose CT: a phantom study. PloS one 10(9), e0138658. https://dx.doi.org/10.1371/journal.pone.0138658

24 O’Neill S, Kavanagh RG, Carey BW, Moore B, Maher M and O’Connor JO. Using body mass index to estimate individualised patient radiation dose in abdominal computed tomography. European Radiology Experimental 2018:2;38 https://doi.org/10.1186/s41747-018-0070-5. 
25 Boos J, Lanzman RS, Heush P, Aissa J, Schleich C, Thomas C et al. Does body mass index outperform body weight asa a surrogate parameter in the calculation of size-specific dose estimates in adult body CT ? BJR 2016;89:20150734. Doi:10.1259/bjr20150734

26 World Health Organization. Obesity: preventing and managing the global epidemic: report a WHO consultation. WHO Technical Report Series 894. 2000

27 Ingenuity TF PET/CT Performance Testing Technical Manual. 459800090571 Revision G, Koninklijke Philips N.V.

28 Philips NM Clinical Science, Enhanced lesion detectability and accurate quantitative imaging, White Paper, Philips Healthcare, USA.

29 Rasband, W.S., ImageJ, U. S. National Institutes of Health, Bethesda, Maryland, USA, https://imagej.nih.gov/ij/, 1997-2018.

30 Kleinbaum DG, Kupper LL and K. E. Muller, Applied Regression Analysis and Other Multivariable Methods PWS-KENT, Boston, Massachusetts, 1988

31 Council Directive 2013/59/Euratom of 5 December 2013 laying down basic safety standards for protection against the dangers arising from exposure to ionising radiation, and repealing Directives 89/618/Euratom, 90/641/Euratom, 96/29/Euratom, 97/43/Euratom and 2003/122/Euratom, 2013. http://data.europa.eu/eli/dir/2013/59/oj 


\section{Figures}

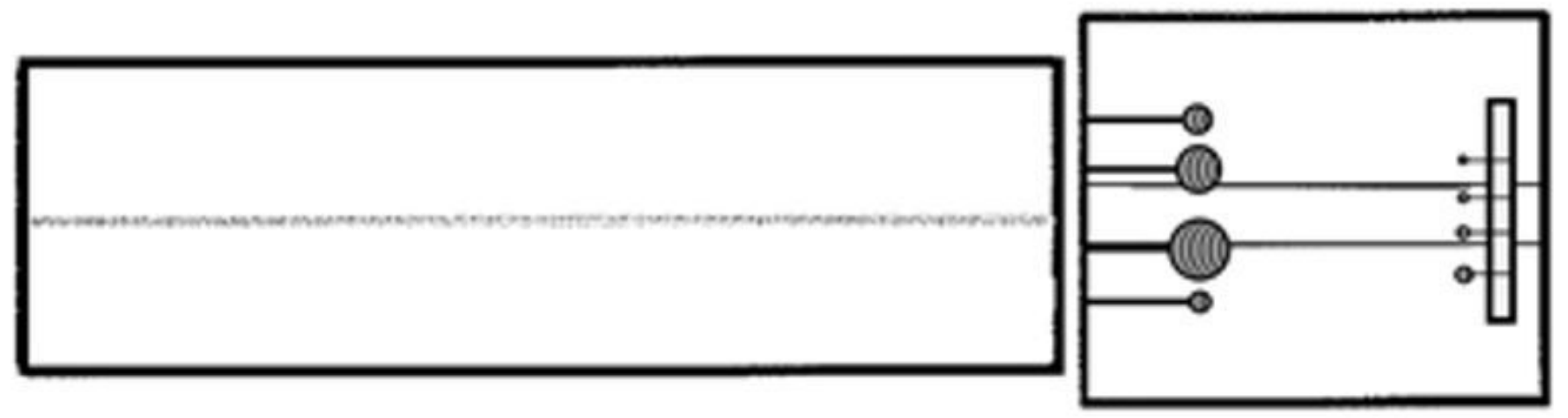

(a)

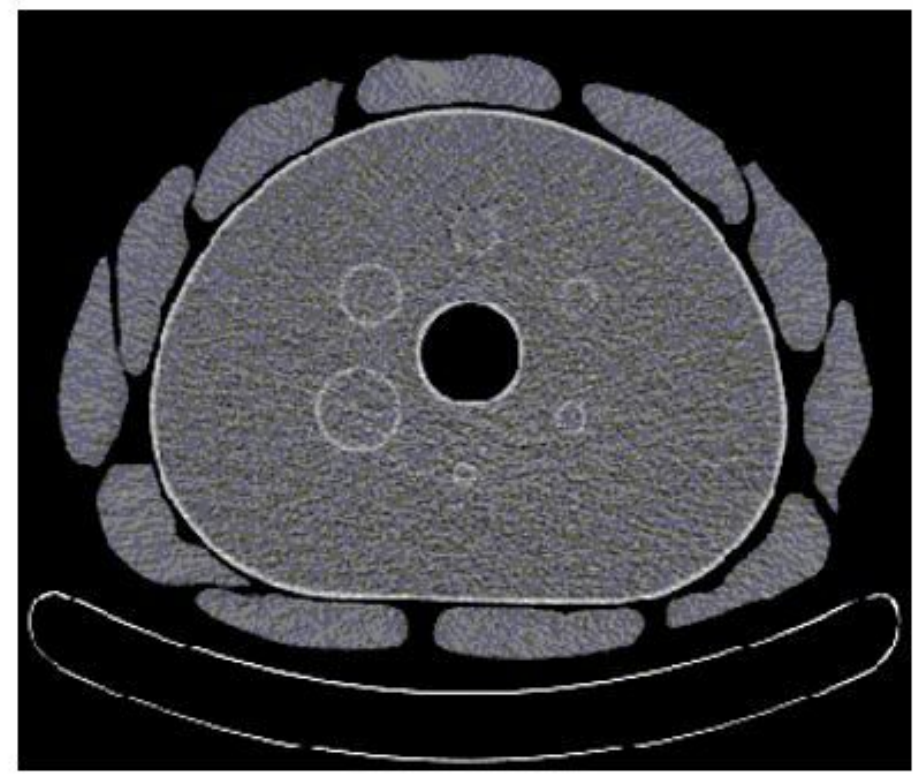

(b)

\section{Figure 1}

Phantom setup in antero-posterior view (a) and CT scan of the b-NEMA-IQ phantom at standard spheres level (b). 
(a)

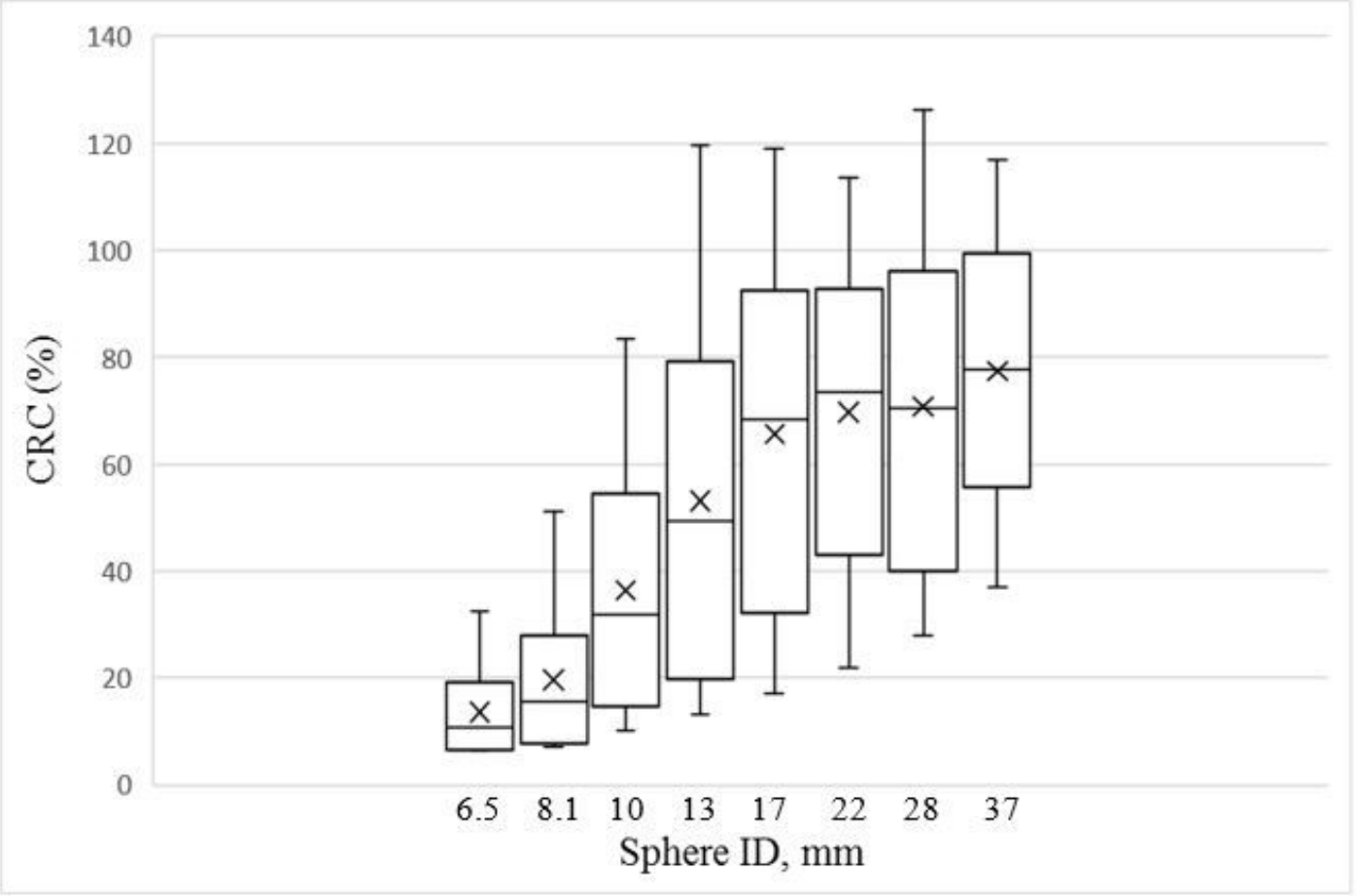

(b)

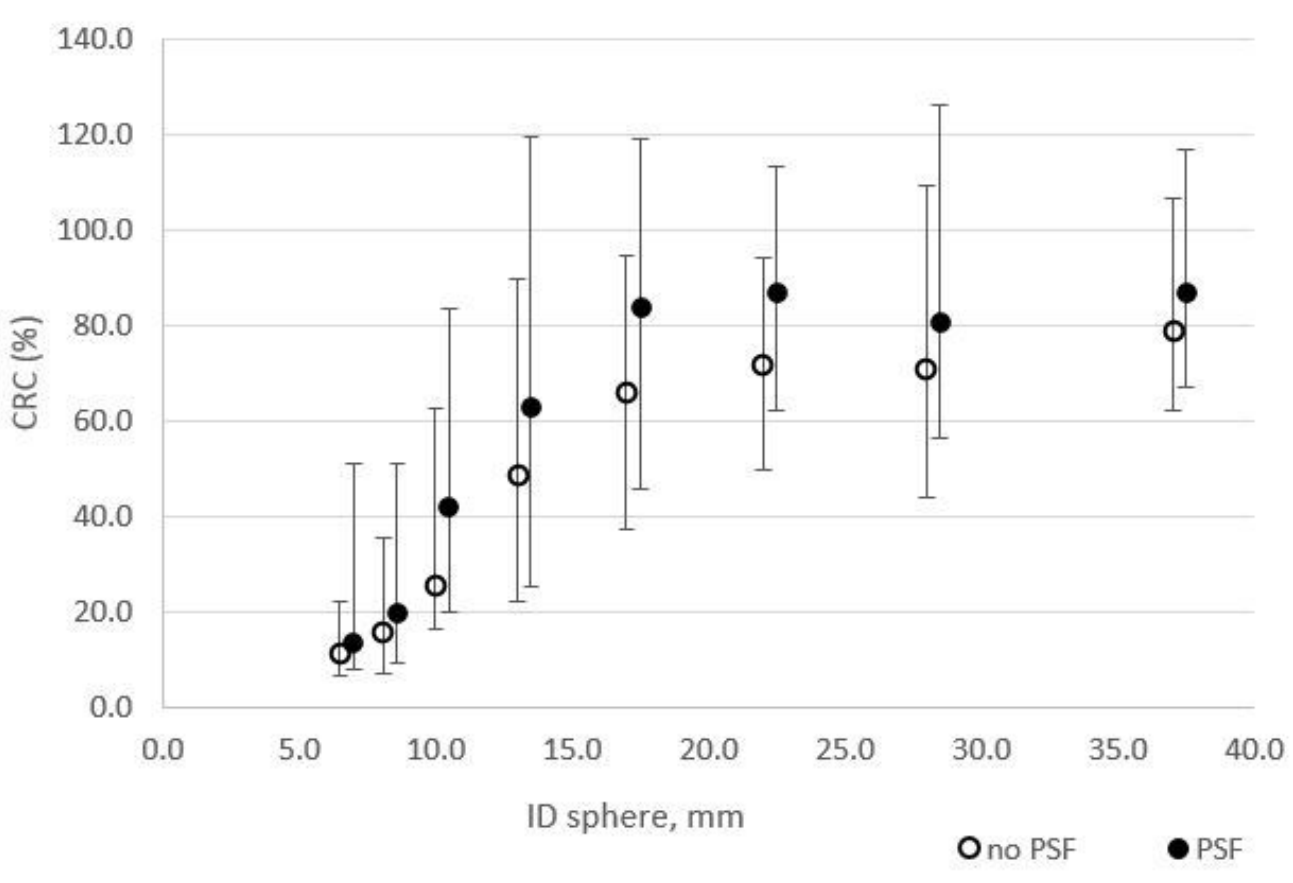

Figure 2

Box plots of CRC values as function of sphere ID (a) and PSF (b). Outliers and extremes are points higher than the value of the 75 th percentile plus 1.5 or 3 times the interquartile distance, or lower than the value of the 25 th percentile minus 1.5 or 3 times the interquartile distance, respectively. 

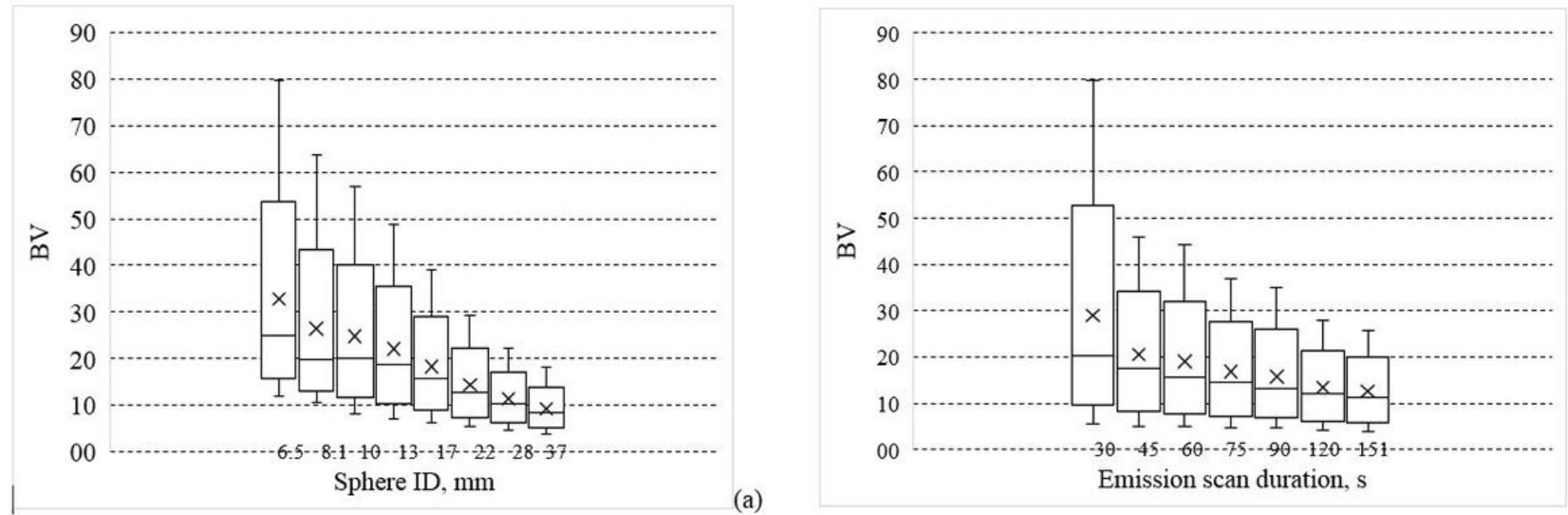

(a)
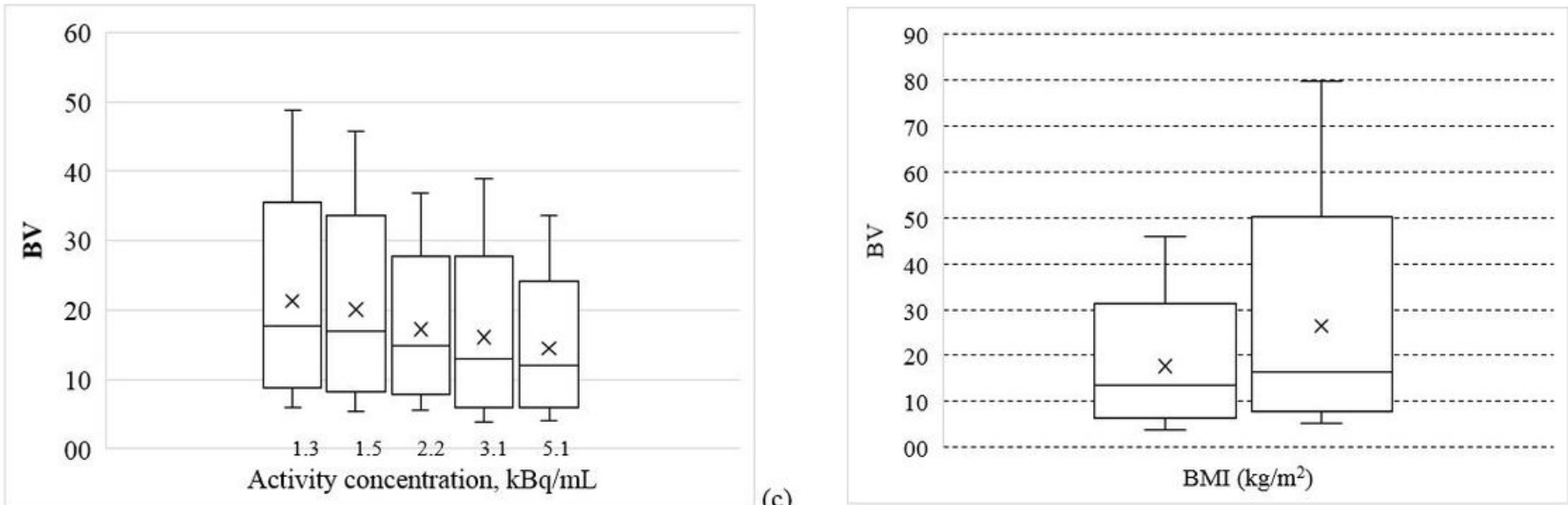

(c)

\section{Figure 3}

Box plots of BV as function of sphere ID (a), ESD (b), AC (c) and W (d). Outliers and extremes are points higher than the value of the 75th percentile plus 1.5 or 3 times the interquartile distance, or lower than the value of the 25 th percentile minus 1.5 or 3 times the interquartile distance, respectively. 

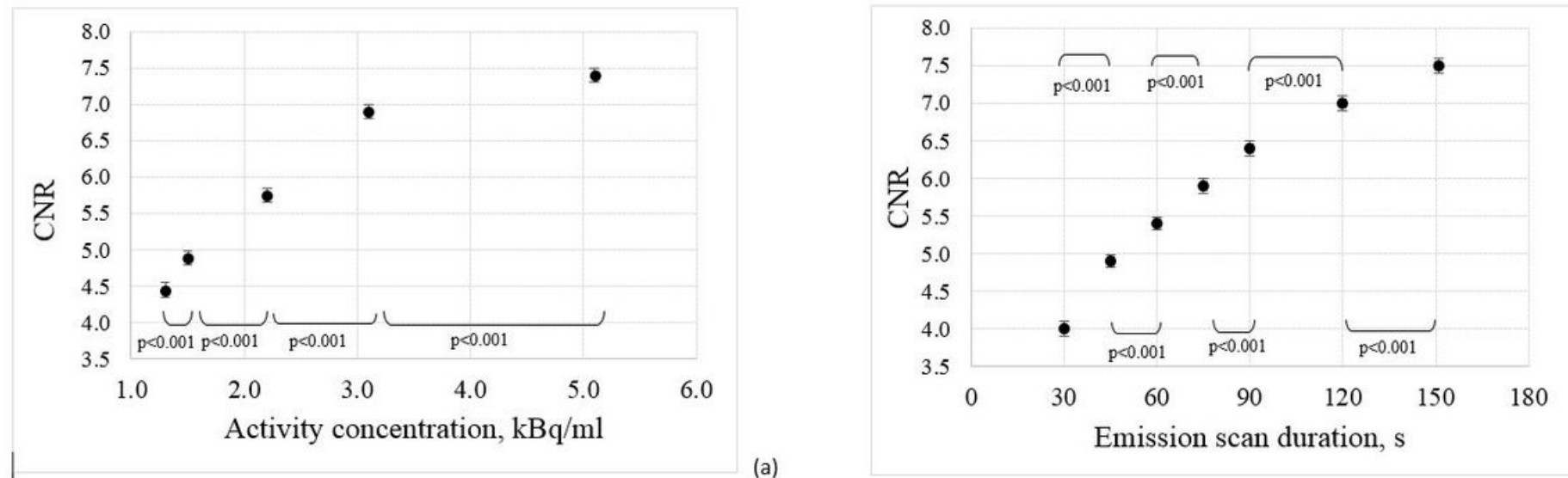

(b)

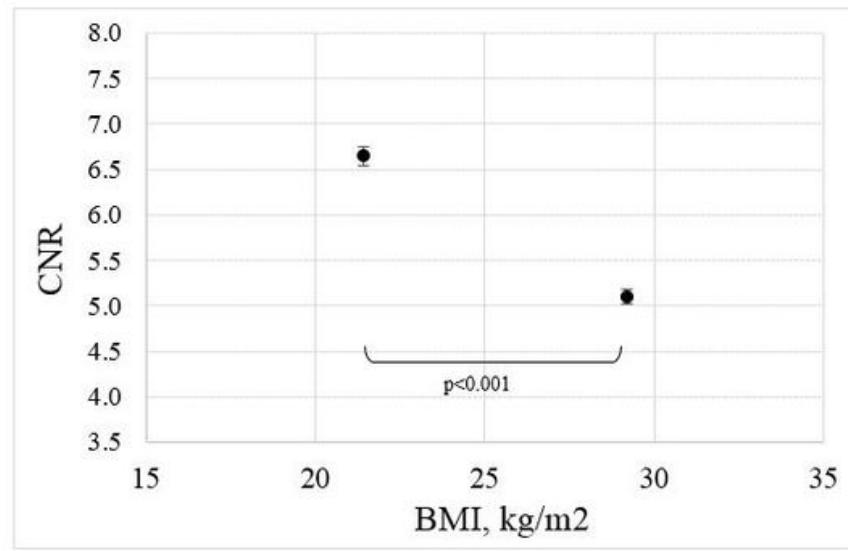

(c)

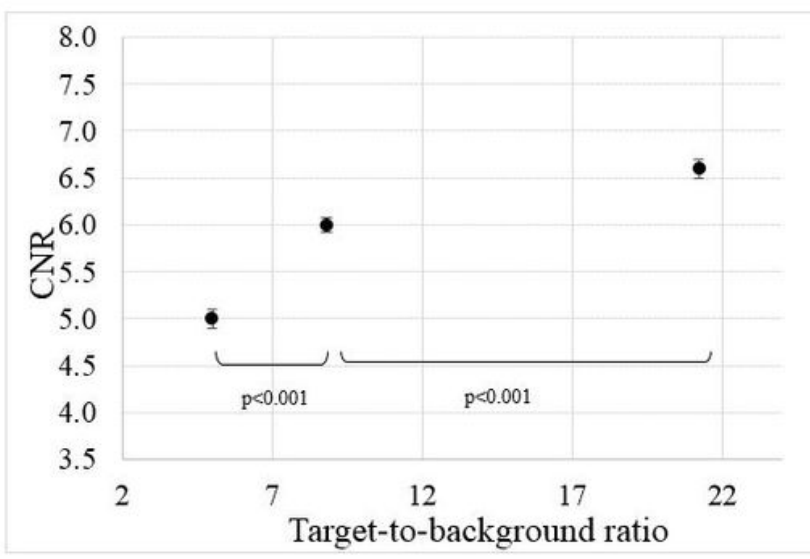

(d)

\section{Figure 4}

CNR as a function of AC (a), ESD (b), W (c) and TBR (d). Points represent least square averages; vertical bars represent $95 \%$ confidence intervals. The results of the Scheffè test are displayed for the adjacent levels of the predictor variables. 

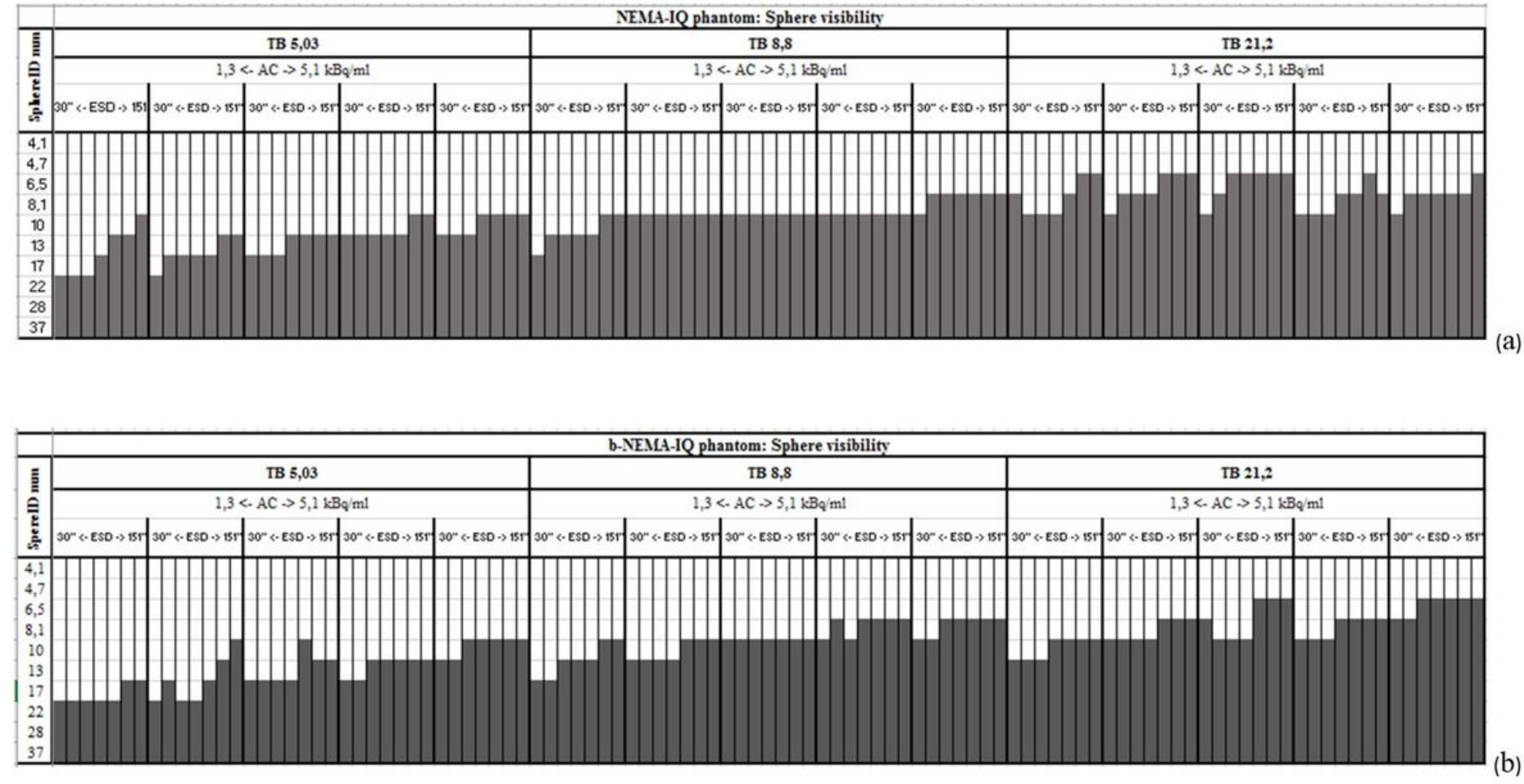

Figure 5

Results of the visual detection of the spheres for NEMA-IQ (a) and b-NEMA-IQ (b) phantoms for different TBR, AC and ESD and spherelD. Grey and white boxes indicate a detectable/undetectable sphere. 
a) ESD
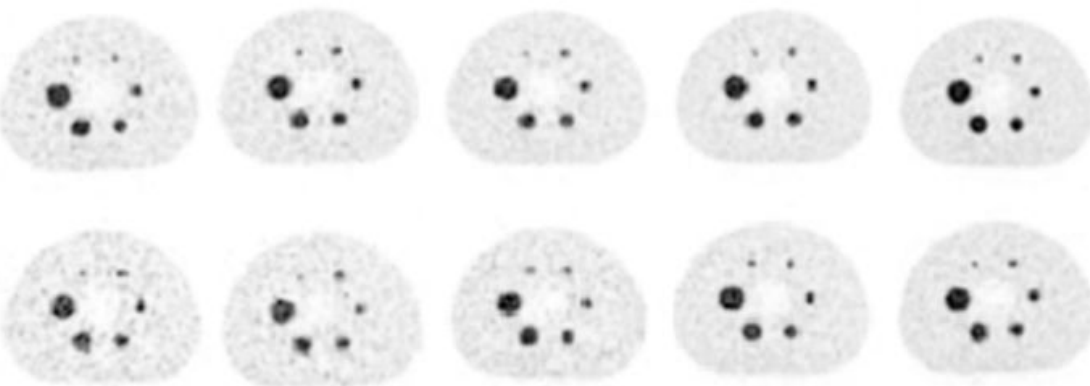

b) $\mathrm{AC}$
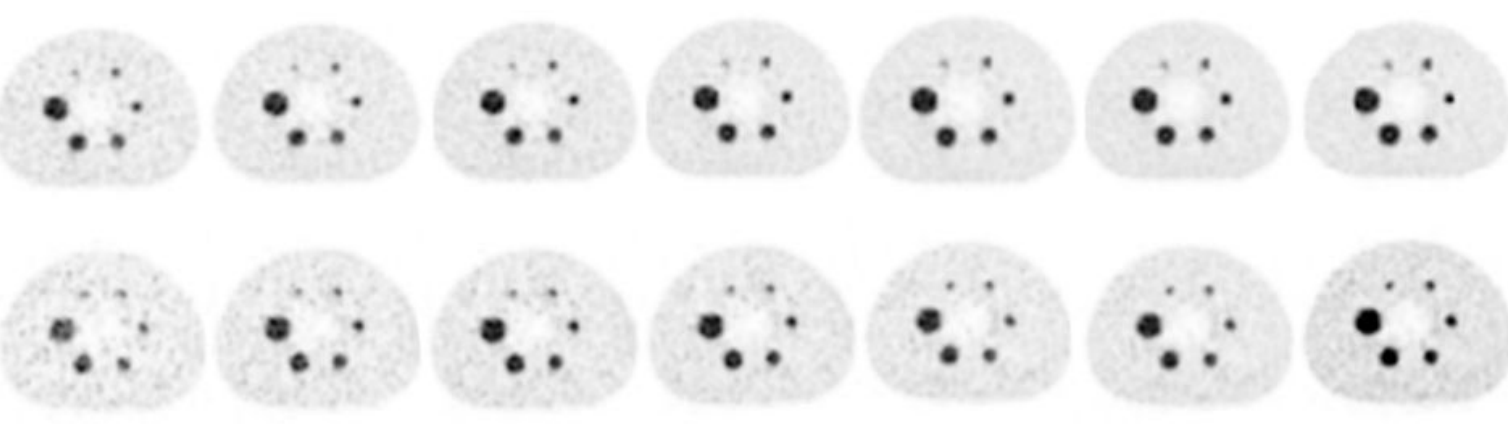

\section{Figure 6}

(a) IEC (upper row) and b-IEC (lower row) images of TBR=9 for increasing AC (from left to right) for $E S D=60$ s. (b) IEC (upper row) and b-IEC (lower row) images of TBR=9 for increasing ESD (from left to right) for $A C=2.0 \mathrm{kBq} / \mathrm{ml}$. The impact of increasing $E S D$ or $A C$ is evident on both the reduction of noise in the background and in the greater visibility of the $10 \mathrm{~mm}$ sphere. 